\title{
Analysis of Inductive Coil Gun Performance Based on Field Coupling Circuit Method
}

\author{
Shoubao Liu, Jiangjun Ruan, Daochun Huang and Zilin Wan \\ School of Electrical Engineering, Wuhan University, Wuhan, 430072 Hubei, China
}

\begin{abstract}
Inductive coil gun's performance analysis is very important for experimental research and its electromagnetic optimization design. Circuit simulation is carried out based on current filament model, the government equations of current filament model may boil down to initial value problem of nonlinear variable coefficient ordinary differential equations, which can be solved by numerical method and is easy to program. From circuit simulation, the dependence of system performance on variable parameter can be found. Field model is built based on composite grid method; composite grid method uses two sets of mutual independent grids to discrete solution region, it overcomes the trouble of remeshing in normal finite element technique based on one set of grid. In this paper performance analysis of three stages coaxial inductive coil gun is carried out based on field coupling circuit method. Three dimensional moving conductor eddy current field simulation of coil gun is realized, field variables distribution in the launching process is obtained. By comparing the results of circuit model and field model the validity of the two models is proved.
\end{abstract}

\section{INTRODUCTION}

Coil launcher is a kind of specific induction motor [1]. Generally, it constitutes by five parts: pulse power, high-speed switch, exciting coil, armature and projectile. The pulse power loads electromagnetic energy on exciting coil after pulsed on. Transient magnetic field produced by the exciting coils induces circumferential eddy current in the armature. The circumferential eddy current interacts with radial component of the magnetic field which produces Lorentz force to drive the armature and projectile in the direction of muzzle. When armature arrives at an appropriate position, the next stage is fired, this process continues until the projectile is out of bore.

Although working principle of coil gun is simple, coil gun's launching is a complex electromagnetic transient process, to obtain main performance parameters and realize optimization design is not easy. Compared with expensive prototype experiments, numerical simulation can save design cost and improve work efficiency greatly in the optimized design of coil gun. The numerical simulation model for coil gun can be divided into two types: circuit model and multi-physical field model [2]. The circuit model has the virtue of simple and easy realization. According to the circuit model, dependence of variable parameters on result can be found easily. The multi-physical field model solves the problem from partial differential equation or integral equation, and can obtain various field quantities accurately, such as magnetic flux density and eddy current density.

In the analysis of coil gun only use circuit model can't get the field distribution, so precise analysis is impossible; only use field model can't complete the whole analysis procedure, since the current of exciting coils which is material property in field model doesn't know in advance. So it is absolutely essential to analyze coil gun based on field and circuit combined model.

\section{BASIC PRINCIPLES OF CIRCUIT MODEL AND FIELD MODEL}

\section{A. Current filament model}

There are two types of circuit model: current sheet model [3] and current filament model (CFM) [4]-[5]. The current sheet model replaces the currents in an actual barrel and in a cylindrical tubular projectile (sleeve) with two equivalent current sheets located at their equivalent radius; by computing the magnetic field produced by the two current sheets the Lorentz force applied on projectile can be obtained. The current filament model divides massive conductive parts of the system into elementary volume elements, in which uniform distribution of current is assumed [6], a current filament is associated with every volume element, and its electrical parameters are calculated, the electrical and mechanical equations governing the behaviors of the system are formulated on the basis of the adopted equivalent network (the schematic diagram of CFM is shown in Fig.1). Considering the definite physical meaning and simple solving process of current filament model, this paper uses it to establish circuit model.

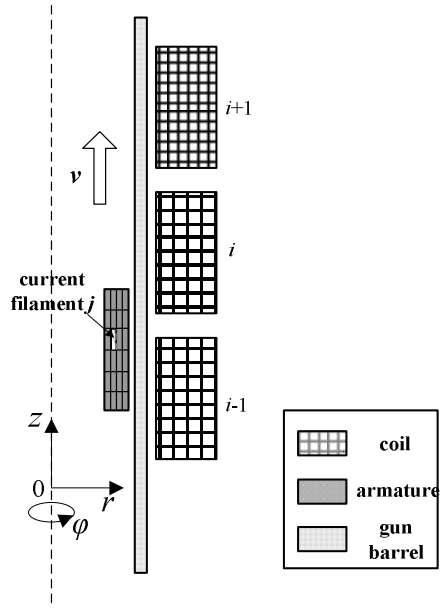

(a) CFM of coil gun 


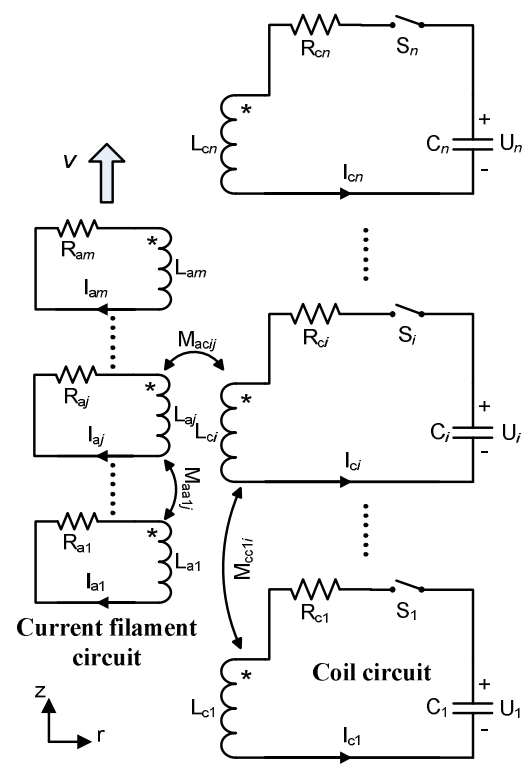

(b) Equivalent circuit diagram of CFM

Fig. 1. Schematic diagram of CFM

As shown in Fig.1(b), there are $n$ stages of existing coils and the armature was divided into $m$ current filaments. According to kirchhoff's voltage law, we can write out the governing equations as

$$
[V]=[R][I]+\frac{d}{d t}\{[L][I]+[M][I]\}
$$

$[V]$ and $[I]$ are column vectors which have $m+n$ elements and denote the voltage vector and current vector, respectively. $[R]$ and $[L]$ are $m+n$ order diagonal matrixes composed of the individual projectile and coil resistances and self-inductances, respectively, and $[M]$ is a square $(m+n) \times(m+n)$ matrix, each element of which represents a mutual inductance between any two individual coils. It should be noted that the mutual inductances $[M]$ between drive coils and projectile coils are functions of the distance which denotes the position of the armature and is a function of time.

Capacitors voltage and coils current satisfy the relations

$$
[C] \frac{d}{d t}\left[V_{c}\right]=-\left[I_{d}\right]
$$

where $[C]$ is a n-order diagonal matrix containing the capacitances. $\left[V_{c}\right]$ and $\left[I_{d}\right]$ are the capacitor voltages and the exciting coils currents column matrices respectively. $\left[V_{c}\right]$ and $\left[I_{d}\right]$ are submatrices of $[V]$ and $[I]$.

The Lorentz force acting on the projectile is given by

$$
F=\frac{1}{2}[I]^{T}[G][I]
$$

where $[G]=d[\mathrm{M}] / d x$ and the superscript $T$ stands for the transpose of the matrix. Finally, combine the equations of motion with (1)-(3). The complete set of government equations which describe the capacitively driven inductive coil gun system is given in matrix form:

$$
\begin{gathered}
\{[L]+[M]\} \frac{d}{d t}[I]= \\
{[V]-[R][I]-v_{p}[G][I]} \\
{[C] \frac{d}{d t}\left[V_{c}\right]=-\left[I_{d}\right]} \\
M_{p} \frac{d v_{p}}{d t}=\sum_{p=1}^{m} \sum_{d=1}^{n} I_{p} I_{d} \frac{d M_{p d}}{d x} \\
\frac{d x}{d t}=v_{p}
\end{gathered}
$$

where $M_{p}$ is the mass of armature and $v_{p}$ is the velocity of armature. So the governing equations represent a set of simultaneous nonlinear differential equations with time-variable coefficients which can be solved by fourth-order Runge-Kutta method. The number of unknown variables in the governing equations is

$$
N=2 n+m+2
$$

$m$ filament currents, $n$ exciting coil currents, $n$ capacitor voltages, $v_{p}$, and $x$

\section{B. Composite Grid Method}

Composite grid method (CGM) developed from the conventional multi-grid method uses two grid sets: one coarse grid for the global region $\left(\Omega_{c}\right)$ and one fine grid for the local region $\left(\Omega_{f}\right), \Gamma=\Omega_{c} \cap \Omega_{f}$ is the interface between the two regions[7], as shown in Fig. 2.

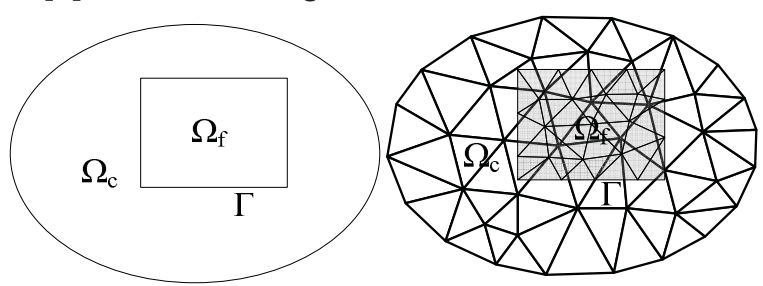

Fig. 2. Solution region and two sets of grids of CGM

The governing equations are written in operator forms below

$$
\begin{cases}L_{c} v=f_{c} & \Omega_{\mathrm{c}} \\ L_{f} u=f_{f} & \Omega_{\mathrm{f}} \\ \left.v\right|_{\Gamma}=\left.u\right|_{\Gamma} & \end{cases}
$$

Where $w$ and $u$ are unknowns, $L_{c}$ and $L_{f}$ denote the operators, $f_{c}$ and $f_{f}$ denote the source items in each region, respectively.

According the principle of virtual work, (9) can be rewritten as

$$
\begin{aligned}
\left(L_{c} v, \bar{v}\right)_{\Omega_{c}} & =\left(f_{c}, \bar{v}\right)_{\Omega_{c}} \\
\left(L_{f} u, \bar{u}\right)_{\Omega_{f}} & =\left(f_{f}, \bar{u}\right)_{\Omega_{f}}
\end{aligned}
$$

By (10) and (11), it is obvious to obtain the following equation

$$
\left(L_{c} v-f_{c}, \bar{v}\right)_{\Omega}=\left(L_{c} v-f_{c}, \bar{v}\right)_{\Omega_{f}}-\left(L_{f} u-f_{f}, \bar{u}\right)_{\Omega_{f}}
$$

The computation scheme in CGM can be written as 


$$
\left(L_{c} v-f_{c}, \bar{v}\right)_{\Omega}=\left(L_{c} u_{c}-f_{c}, \bar{v}\right)_{\Omega_{f}}-\left(L_{f} u_{f}-f_{f}, \bar{u}\right)_{\Omega_{f}}
$$

where $u_{c}$ and $u_{f}$ denote the variable in $\Omega_{f}$ associated with the operators $L_{c}$ and $L_{f}$ respectively.

The basis functions in the global and local grids are $\Psi=\left\{\Psi_{j}\right\}_{j=1}^{n_{c}}$ and $\Phi=\left\{\Phi_{j}\right\}_{j=1}^{n_{f}}$, where $n_{c}$ and $n_{f}$ are the numbers of nodes in each set of grids. Variable $u, v, \bar{u}$ and $\bar{v}$ can be expressed as

$$
\begin{array}{ll}
v=\sum_{j=1}^{n_{c}} v_{j} \Psi_{j}=\boldsymbol{V}^{T} \boldsymbol{\Psi}, & \bar{v}=\sum_{j=1}^{n_{c}} \bar{v}_{j} \Psi_{j}=\overline{\boldsymbol{V}}^{T} \boldsymbol{\Psi} \\
u=\sum_{j=1}^{n_{f}} u_{j} \Phi_{j}=\boldsymbol{U}^{T} \boldsymbol{\Phi}, & \bar{u}=\sum_{j=1}^{n_{f}} \bar{u}_{j} \boldsymbol{\Phi}_{j}=\overline{\boldsymbol{U}}^{T} \boldsymbol{\Phi}
\end{array}
$$

Define a matrix $\boldsymbol{D}$ as

$$
\Psi=D \Phi
$$

Equation (16) develops the mapping relation between the global and local grids. It is satisfied in $\Omega_{f}$ that

$$
v=\boldsymbol{V}^{T} \boldsymbol{D} \boldsymbol{\Phi}
$$

Equation (13) can be expanded as

$$
\begin{gathered}
\int_{\Omega} \boldsymbol{\Psi} L_{c}\left(\boldsymbol{\Psi}^{T}\right) d \Omega \boldsymbol{V}=\int_{\Omega} f_{c} \boldsymbol{\Psi} d \boldsymbol{\Omega}+\boldsymbol{D}\left(\boldsymbol{R}_{c}-\boldsymbol{R}_{f}\right) \\
\boldsymbol{R}_{c}=\int_{\Omega_{f}} \boldsymbol{\Phi} L_{c}\left(\boldsymbol{\Phi}^{T}\right) d \Omega \boldsymbol{U}_{c}-\int_{\Omega_{f}} \boldsymbol{\Phi} f_{c} d \Omega \\
\boldsymbol{R}_{f}=\int_{\Omega_{f}} \boldsymbol{\Phi} L_{f}\left(\boldsymbol{\Phi}^{T}\right) d \Omega \boldsymbol{U}_{f}-\int_{\Omega_{f}} \boldsymbol{\Phi} f_{f} d \boldsymbol{\Omega}
\end{gathered}
$$

The correction term $\boldsymbol{R}_{c}-\boldsymbol{R}_{f}$ is calculated in the local grid. The addition of this local grid term to the equation of global grid is realized by multiplying a $\boldsymbol{D}$ matrix in front of it

The nodal result $\boldsymbol{U}_{c}$ and $\boldsymbol{U}_{f}$ are the solutions of two Dirichlet boundary value problems in $\Omega_{f}$.

$$
\begin{aligned}
\left(L_{c} u_{c}, \bar{u}\right)_{\Omega_{f}} & =\left(f_{c}, \bar{u}\right)_{\Omega_{f}} \\
\left(L_{f} u_{f}, \bar{u}\right)_{\Omega_{f}} & =\left(f_{f}, \bar{u}\right)_{\Omega_{f}}
\end{aligned}
$$

It should be pointed out that the Dirichlet boundary condition is imposed to the matrix equation in finite element procedure. The values of $\boldsymbol{R}_{c}$ and $\boldsymbol{R}_{f}$ are non-zero at the degrees of freedom belonging to the Dirichlet boundary.

The Dirichlet boundary conditions of (21) and (22) are interpolated through the results calculated in the global grid. continuity boundary condition in (9) is weakly satisfied by

$$
(u-v, \bar{u})_{\Gamma}=0
$$

which can be written as

$$
\overline{\boldsymbol{U}}^{T} \int_{\Gamma} \boldsymbol{\Phi} \boldsymbol{\Phi}^{T} d \Gamma \boldsymbol{U}=\overline{\boldsymbol{U}}^{T} \int_{\Gamma} \boldsymbol{\Phi} \boldsymbol{\Phi}^{T} d \Gamma \boldsymbol{D}^{T} \boldsymbol{V}
$$

So the mapping from global grid to local grid is through the relation

$$
\boldsymbol{U}=\boldsymbol{D}^{T} \boldsymbol{V}
$$

The calculation procedure of CGM is shown in Fig. 3, the iteration continues until the results' convergence is achieved in every time-step. Since the two grid sets are created independently, CGM overcomes the trouble caused by only one set of grid and it is very convenient to handle with MCEC problems [8].

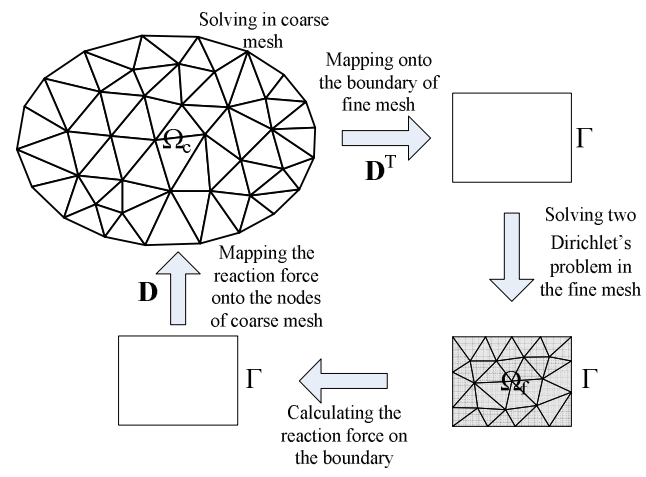

Fig. 3. Calculation procedure of CGM

\section{Couple the Two Method In AnAlysis of CoIL GuN}

In order to couple the two methods in the analysis of coil gun, a three-stage coaxial inductive coil gun simulation model is built. The parameters of exciting coils and armature are listed in table I.

TABLE I

PARAMETERS OF EXCITING COILS AND ARMATURE

\begin{tabular}{|c|c|c|}
\hline \multirow{4}{*}{ Coil } & Inside radius & $80 \mathrm{~mm}$ \\
\cline { 2 - 3 } & Radial thickness & $50 \mathrm{~mm}$ \\
\cline { 2 - 3 } & Axial length & $107 \mathrm{~mm}$ \\
\cline { 2 - 3 } & Spacing distance & $28 \mathrm{~mm}$ \\
\cline { 2 - 3 } & Turn number of wire & 38 \\
\hline \multirow{5}{*}{ Armature } & Inside radius & $55 \mathrm{~mm}$ \\
\cline { 2 - 3 } & Radial thickness & $15 \mathrm{~mm}$ \\
\cline { 2 - 3 } & Axial length & $103 \mathrm{~mm}$ \\
\cline { 2 - 3 } & Mass & $1 \mathrm{~kg}$ \\
\cline { 2 - 3 } & Conductivity & $3.54 \times 10^{7} \mathrm{~S} / \mathrm{m}$ \\
\cline { 2 - 3 } & Differential permeability & 1 \\
\cline { 2 - 3 } & Initial velocity & $0 \mathrm{~m} / \mathrm{s}$ \\
\hline
\end{tabular}

The pulse capacitors' parameters are listed in table II . TABLE

PARAMETERS OF CAPACITOR

\begin{tabular}{|c|c|c|}
\hline Capacitor number & Capacitance $(\mathrm{mF})$ & Charge voltage $(\mathrm{kV})$ \\
\hline 1 & 20.485 & 4.6 \\
\hline 2 & 5.863 & 9.1 \\
\hline 3 & 2.059 & 14.7 \\
\hline
\end{tabular}

At first we use circuit model to calculate some parameters in the launching process, such as currents of each coil, force acting on the armature, velocity of armature. The structure diagram of coil gun and division of current filament is shown in Fig. 4. Current waveform of exciting coils and current filaments obtained from circuit simulation are shown in Fig. 5.

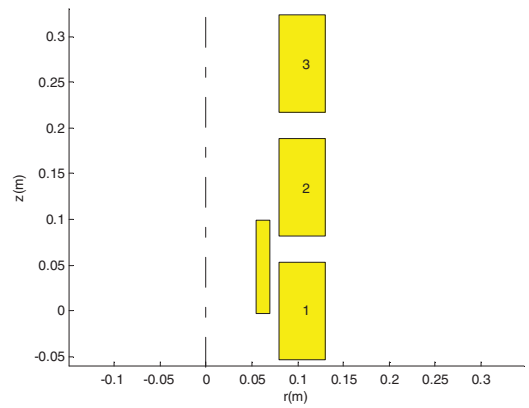

(a) Structure diagram of three-stage coil gun 


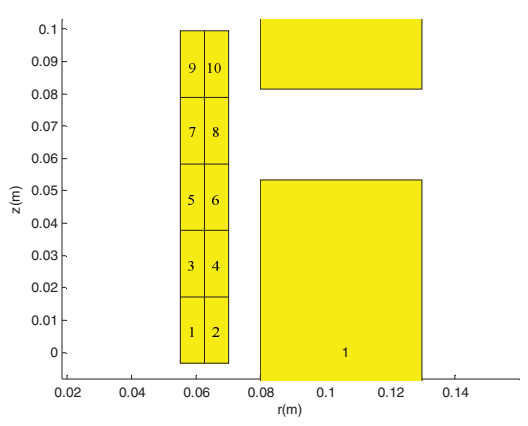

(b) Division of current filament

Fig. 4. Structure diagram of coil gun and division of current filament

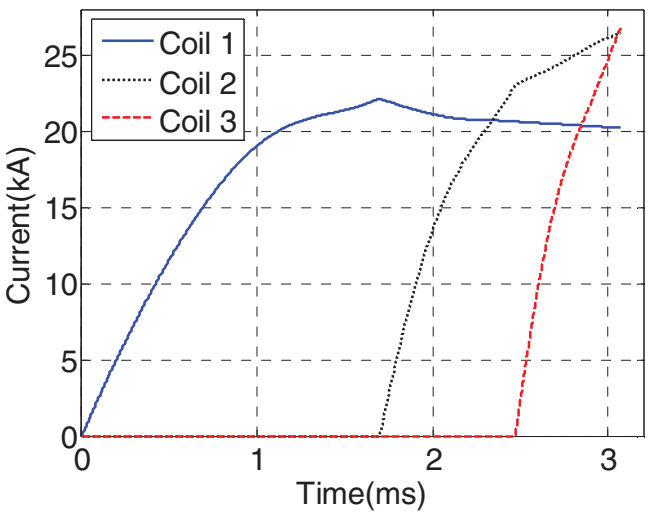

(a) Current of exciting coil

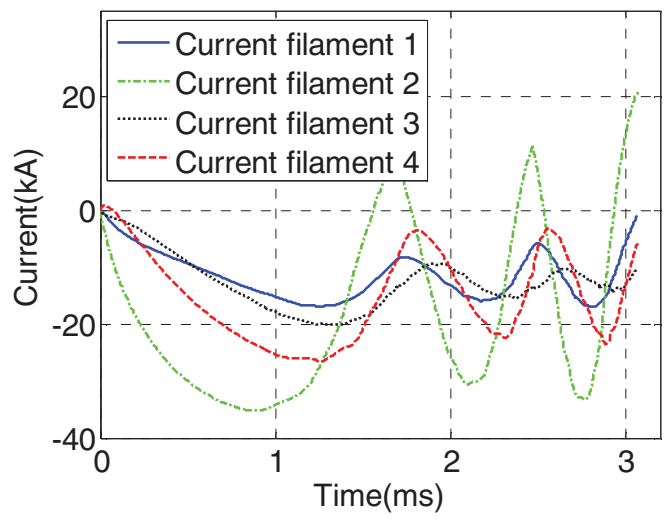

(b) Current of the first four current filaments

Fig. 5. Current waveform of circuit model

Then the 3D field model based on CGM is built as shown in Fig. 6. The currents got by circuit model are loaded in field model as material properties of exciting coils . By solving the field model field quantities such as eddy current field, magnetic flux density at different time can be obtained (as displayed in Fig.7 and Fig.8).
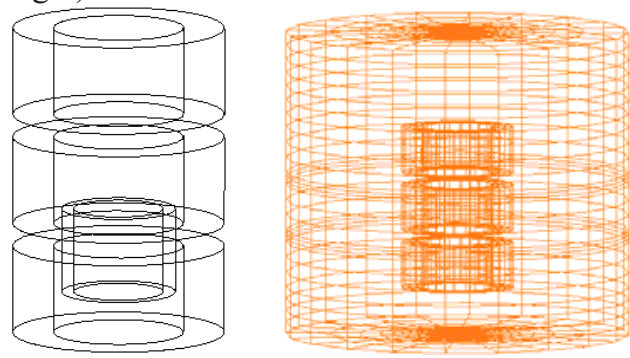

(a) $3 \mathrm{D}$ views

(b) Global region meshed in coarse grids

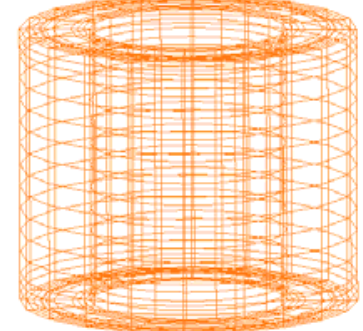

(c) Armature meshed in fine grids

Fig. 6. Field model and domain mesh

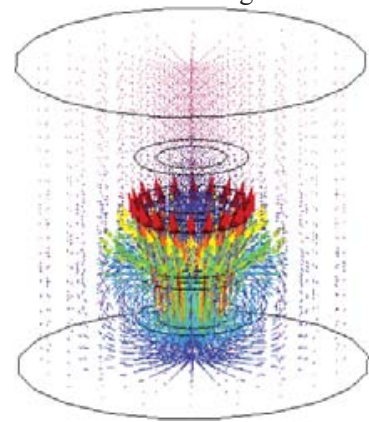

(a) Flux density in coarse mesh

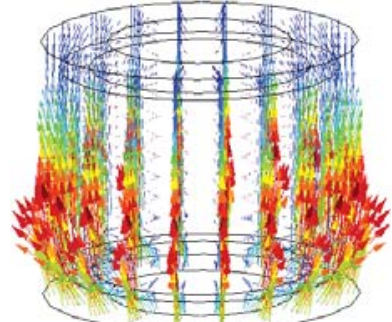

(b) Flux density in armature

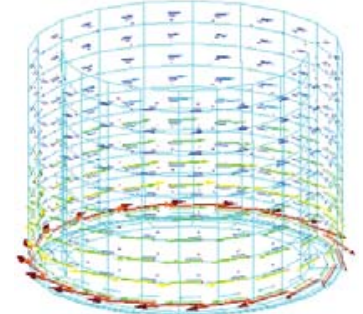

(c) Eddy in armature

Fig. 7. Field quantities distribution at $0.60 \mathrm{~ms}$

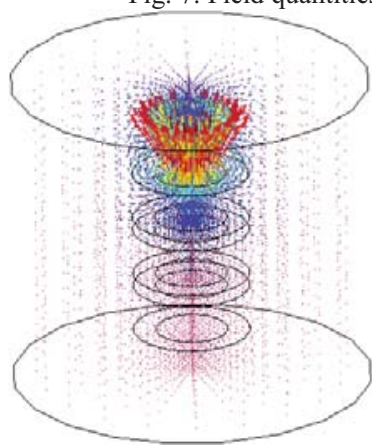

(a) Flux density in coarse mesh

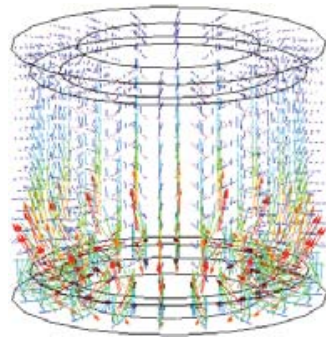

(b) Flux density in armature

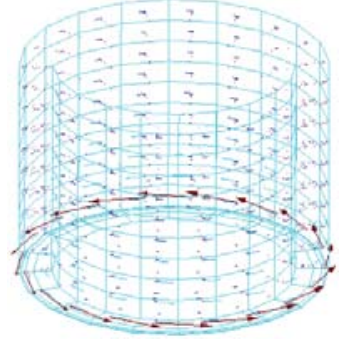

(c) Eddy in armature

Fig. 8. Field quantities distribution at $2.96 \mathrm{~ms}$

From Fig. 7 and Fig. 8, we can see that traveling magnetic field produced by exciting coils moves ahead with armature. The induced eddy current mainly distributes in the back end of the armature which shows agreement with circuit model (as 
shown in Fig.5 (b)). Results of the two models are compared (as shown in Fig. 9) and the validity of the coupling method is proved.

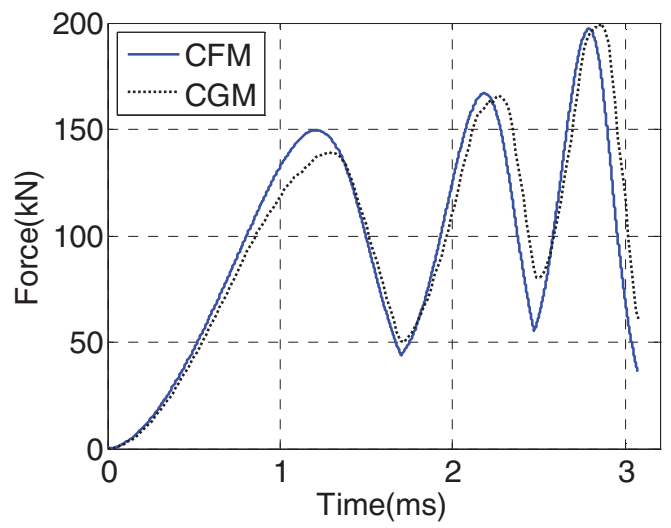

(a) Force curve of CFM and CGM

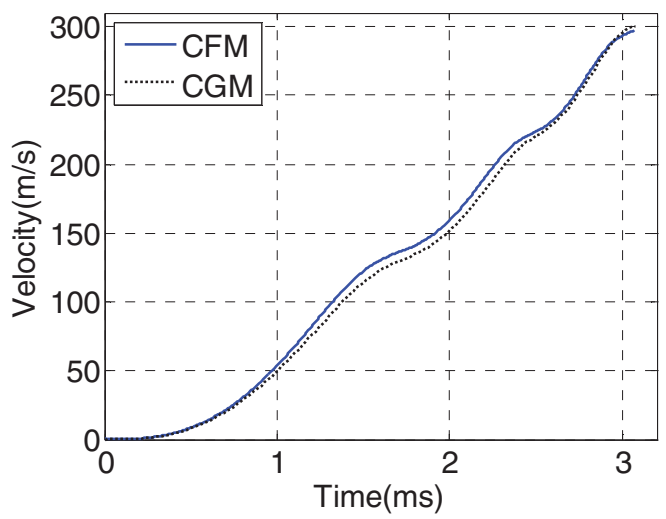

(b) Velocity result of CFM and CGM

Fig. 9. Results comparison of circuit model and field model

\section{CONCLUSION}

This paper described a kind of specific induction motor that is inductive coil gun, introduced its circuit model based on current filament method and field model based on composite grid method, built a three-stage coaxial inductive coil gun and simulated it according to "field-circuit" combined technique. By comparing the results obtained by the two methods the validity of field combined circuit method is proved. The method presented in this paper is very important and useful for precise design of inductive coil gun.

\section{ACKNOWLEDGMENT}

The authors would like to thank Dr Zhang Yu for his study on the principle of CGM and Dr Peng Ying for his suggestion on coil gun circuit simulation.

\section{REFERENCES}

[1] R Haghmaram., A Shoulaie, "Literature review of theory and technology of air-core tubular linear induction motors [electromagnetic launcher applications]," Universities Power Engineering Conference 2004, pp. 517-5221.

[2] M. S. Aubuchon, T. R. Lockner, B. N.Turman et al, "Results from Sandia National Laboratories / Lockheed Martin Electromagnetic Missile Launcher (EMML)," IEEE Pulsed Power Conference 2005, 2005:75-78.
[3] J. L He., E. Levi, Z. Zabar, L. Birenbaum and Y. Naot, "Analysis of induction-type coilgun performance based on cylindrical current sheet model," IEEE Transactions on magnetics, vol. 27, no. 1, pp. 579-584, Jan.1991.

[4] Jianliang He, Enrico Levi, Zivan Zabar and Leo Birenbaum, "Concering the design of capacitively driven induction coil guns," IEEE Transactions on magnetics, vol. 17, no. 3, pp. 429-438, Jun. 1989.

[5] Ronald J. Kaye, “ Operational requirements and issues for coilgun electromagnetic launchers," IEEE Transactions on magnetics, vol. 41, no. 1, pp. 194-199, Jan. 2007.

[6] David G. Elliott, "Mesh-matrix analysis method for electromagnetic launchers," IEEE Transactions on magnetics, vol. 25, no. 1, pp. 164-169, Jan. 1989.

[7] Peng Ying, Ruan Jiangjun, Zhang Yu and Gan Yan. "A composite grid method for moving conductor eddy-current problem," IEEE Transactions on magnetics, vol. 43, no. 7, pp. 3259-3265, Jul. 2007.

[8] Zhang Yu, Ruan Jiangjun, Gan Yan, Peng Ying and Du Zhiye. "Application of a Composite Grid Method in the Analysis of 3-D Eddy Current Field Involving Movement," IEEE Transactions on magnetics, vol. 44, no. 6, pp. 1298-1301, Jun. 2008. 